\title{
REVIEW
}

\section{Optical second-harmonic generation measurement for probing organic device operation}

\author{
Takaaki Manaka and Mitsumasa Iwamoto
}

We give a brief overview of the electric-field induced optical second-harmonic generation (EFISHG) technique that has been used to study the complex behaviors of organic-based devices. By analyzing EFISHG images of organic field-effect transistors, the in-plane two-dimensional distribution of the electric field in the channel can be evaluated. The susceptibility tensor of the organic semiconductor layer and the polarization of the incident light are considered to determine the electric field distribution. EFISHG imaging can effectively evaluate the distribution of the vectorial electric field in organic films by selecting a light polarization. With the time-resolved technique, measurement of the electric field originating from the injected carriers allows direct probing of the carrier motion under device operation, because the transient change of the electric field distribution reflects the carrier motion. Some applications of the EFISHG technique to organic electronic devices are reviewed.

Light: Science \& Applications (2016) 5, e16040; doi:10.1038/Isa.2016.40; published online 11 March 2016

Keywords: electric field; optical second-harmonic generation; semiconductor device

\section{INTRODUCTION}

In recent decades, organic devices such as organic light emitting diodes (OLEDs), organic field effect transistors (OFETs), and organic solar cells (OSCs) have drawn attention because of the great advantages of organic semiconductors ${ }^{1}$. The fabrication of organic devices using cost-effective process such as ink-jet and roll-to-roll flexographic printing is of particular interest. The device characteristics have been improved with the development of the fabrication process and highperformance materials ${ }^{2}$. However, the operation mechanism of organic devices has not been sufficiently elucidated. Understanding of the device physics is important for the further improvement of device performance.

For silicon-based devices, sophisticated experimental techniques to evaluate the device operation have been available for a long time, and the physical model of the device has been well established. Thus, attempts can be made to understand the organic devices on the basis of such experimental techniques and device physics developed for the inorganic devices. For instance, the development of the scanning probe microscope technique makes it possible to reveal the potential distribution in inorganic devices such as metal-oxide semiconductor $\mathrm{FETs}^{3-6}$. These results motivated us to use a scanning Kelvin probe to visualize the local potential in OFETs. Bürgi et al. ${ }^{7}$ have successfully revealed the potential distribution in the channel of OFETs. Based on the high spatial resolution of the probe microscope technique, a potential fluctuation in a pentacene OFET with a polycrystalline active layer has also been examined using atomic force microscope potentiometry ${ }^{8}$.
The majority of the carriers in silicon-based devices are supplied and precisely controlled by the impurity doping process. Carriers in the silicon-based devices are governed by the Fermi-Dirac statistics, in which thermal equilibrium is established over the entire device, and the local carrier density is governed by the local electric potential. Thus, evaluation of the potential distribution is important when discussing the device operation in silicon-based devices. However, precise control of the same impurity doping process in organic semiconductors is difficult because the doping mechanism differs from that for silicon single crystals. The chemical dopant is unstable, and an appropriate dopant has not been developed. Further, typical organic semiconductors, for example, pentacene, have initially low carrier density, which leads to a long dielectric relaxation time. Consequently, the dielectric properties of the organic semiconductor layer should be considered when discussing the device operation.

Recently, optical-based techniques have been extensively developed to investigate organic devices. For instance, charge modulation spectroscopy was developed to investigate the carrier behavior in organic devices ${ }^{9,10}$. The charge modulation technique in the infrared region is also a powerful tool that can be used to understand the microscopic characteristics of carriers, such as polaron formation. Charge-modulated reflectance imaging has been demonstrated to directly visualize the injected carrier distribution in OFETs ${ }^{11}$. Nonlinear optical (NLO) techniques are also effective in studying organic devices. The ultrafast charge carrier dynamics at the interface between the copper-phthalocyanine $(\mathrm{CuPc})$ and $\mathrm{C}_{60}$ layers has been studied 
using time-resolved second-harmonic generation $(\mathrm{SHG})^{12}$. Sum frequency generation (SFG) spectroscopy has also been used to observe the device operation of OFETs ${ }^{13,14}$. We have also developed the electric field-induced SHG (EFISHG) technique to investigate organic devices. As mentioned, the evaluation of electric potential is important in Si-based devices. In contrast, evaluation of the electric field is principally important in studying the operation of organic devices because the electric field governs the carrier processes. Carrier injection is directly influenced by the electric field, and the transport process is governed by the space charge field, as seen in Child's law ${ }^{15}$. Beyond these points, a basic current equation, $j=e n \mu E$, where $e$ and $n$ represent elementary charge and the carrier density, respectively, and $\mu$ is the mobility, also indicates that the electric field plays a significant role in carrier transport.

Mapping of the electric field has been conducted on the basis of optical measurements. Morris et al. ${ }^{16}$ have attempted to map the electric field distributions in operating lateral bulk heterojunctions. Their experimental design is quite similar to our technique, although they used transparent geometry. They directly observed the change in the electric field distribution after laser exposure. Lanzani has reported the mapping of the electric field in fluorinated copper phthalocyanine FET on the basis of Stark spectroscopy ${ }^{17}$. The electro-reflectance (ER) response from the organic semiconductor layer is proportional to the electric field. A confocal setup allows improvement of the lateral resolution (better than $500 \mathrm{~nm}$ ). For the EFISHG measurement, the signal intensity is directly governed by the nonlinear optical susceptibility, whereas the ER response is not greatly affected by the nonlinear optical susceptibility. However, the absorption peak should be located in the spectral region of the ER measurement.

In this review, we briefly summarize our developed method for imaging the electric field in electronic devices on the basis of the EFISHG measurement. This technique provides a new way to understand the carrier behaviors in organic and inorganic devices ${ }^{18,19}$. By applying this technique, the distribution of the electric field in the OFET channel is visualized with high spatial resolution ${ }^{20,21}$. The evaluated distribution of the electric field is in good agreement with the field distribution obtained from the Laplace equation ${ }^{22}$. Taking into account the vectorial nature of the electric field, the in-plane electric field distribution evaluated by the SHG measurement is discussed for OFETs composed of a $\mathrm{C}_{60}$ active layer in this paper. Because the electric field is a vectorial quantity, both the direction of the electric field and its magnitude are significant in detailed investigations of the carrier behavior in organic devices. Because of the coherent process of the SHG, the signal intensity depends on the direction of the static electric field and the nonlinear optical susceptibility tensor. Therefore, understanding the relationship between the direction of the static electric field and the polarization of the SHG signal is important in using the EFISHG technique to probe the electric field. Then, we show that the carrier motion in OFET is captured by direct coupling with the time-resolved technique. Carrier mobility is evaluated by analyzing the transient behavior of the injected carrier.

\section{BASICS OF THE EFISHG}

Let us begin with the brief theory of the EFISHG. Nonlinear optical polarization due to the EFISHG process is expressed as

$$
P_{i}^{2 \omega}=\chi_{i j k}^{(2)} E_{j}^{\omega} E_{k}^{(\omega)}+\chi_{i j k l}^{(3)} E_{j}^{0} E_{k}^{\omega} E_{l}^{(\omega)}
$$

where $E^{0}$ presents the static electric field applied to the material and $E^{\omega}$ is the electric field of light. This polarization produces the frequency-doubled light of the fundamental light under the static electric field. Here, we separately describe the contributions from the orientational polarization and the electronic polarization of molecules. Thus, $\chi_{i j k}^{(2)}$ and $\chi_{i j k l}^{(3)}$ represent the second- and third-order NLO susceptibility tensors, respectively. The indices $j k l$ refer to the Cartesian components of the electric field. When we apply the static electric field to the solution of dipolar molecules, these molecules align in accordance with the external electric field. As a result, an SHG signal is induced because the centrosymmetry of the molecular arrangement is broken. This phenomenon is an effective way to evaluate the second-order molecular hyperpolarizability ${ }^{23,24}$. We can also analyze the side-chain orientation in the poled polymers by SHG. Poled polymers are an important candidate for fabricating electro-optical devices, in which polymer side chains align to the electric field in the glass state of polymers. The poled structure is necessary to obtain the electro-optical functions. For both cases, the first term of Equation (1) is the major origin of the SHG. However, electronic polarization dominates the induced polarization if the constituent molecules possess centrosymmetry. In such cases, the external electric field slightly distorts the electron distribution in a molecule, and effective polarization is induced. Centrosymmetry is broken by the external electric field, and the SHG signal will be generated from the material with inversion symmetry. In other words, a centrosymmetric molecule initially has no molecular second-order hyperpolarizability, but the effective hyperpolarizability is induced under the external electric field. The latter EFISHG represents the third-order NLO processes in a broad sense, because the thirdorder nonlinear optical susceptibility $\chi_{i j k l}^{(3)}$ describes the EFISHG process.

The nonlinear optical susceptibilities are related to the ensemble average of the molecular hyperpolarizabilities as $\chi_{i j k l}^{(3)}=N\left\langle T_{i j k l}^{\mu v \xi o}\right\rangle \gamma_{\mu v \xi o}$, where $N$ and $\gamma_{\mu \nu \xi \xi o}$ are the volume density of molecules and the third-order molecular hyperpolarizability, respectively. $T_{i j k l}^{\mu v \xi o}$ represents the transformation matrix between the molecular coordinate and the laboratory coordinate, and is expressed conventionally using the Euler rotation matrix ${ }^{25}$. The delimiters \langle\rangle denote an ensemble average over the molecular orientations and $\left\langle T_{i j k l}^{\mu v \xi o}\right\rangle$ is expressed explicitly using the orientational distribution function $f(\phi, \beta, \varphi)$ as

$$
\left\langle T_{i j k l}^{\mu \nu \xi o}\right\rangle=\frac{\int_{0}^{2 \pi} \int_{0}^{\pi} \int_{0}^{2 \pi} T_{i j k l}^{\mu v \xi o} f(\phi, \beta, \varphi) \sin \beta d \phi d \beta d \varphi}{\int_{0}^{2 \pi} \int_{0}^{\pi} \int_{0}^{2 \pi} f(\phi, \beta, \varphi) \sin \beta d \phi d \beta d \varphi}
$$

where $\phi, \beta$, and $\varphi$ represent Euler angles between the molecular coordinate and the laboratory coordinate. The complexity of the above calculation can be reduced depending on the structure of the films, that is, the symmetry of the films. If the film is amorphous or consists of randomly-oriented molecules, we can consider $f(\phi, \beta, \varphi)=1$.

Assuming that the macroscopic symmetry of the film is classified into $C_{\infty v}$, the number of independent components of the susceptibility tensor is reduced to eight, that is, $\chi_{1111}=\chi_{2222}$, $\chi_{1122}=\chi_{2211}, \quad \chi_{1122}=\chi_{2233}, \quad \chi_{1212}=\chi_{1221}=\chi_{2112}=\chi_{2121}, \quad \chi_{1313}=$ $\chi_{1331}=\chi_{2332}=\chi_{2323}, \chi_{3113}=\chi_{3223}, \chi_{3311}=\chi_{3322}$ and $\chi_{3333}{ }^{26}$, where the coordinate system is defined in Figure 1c. Therefore, the nonlinear 
a
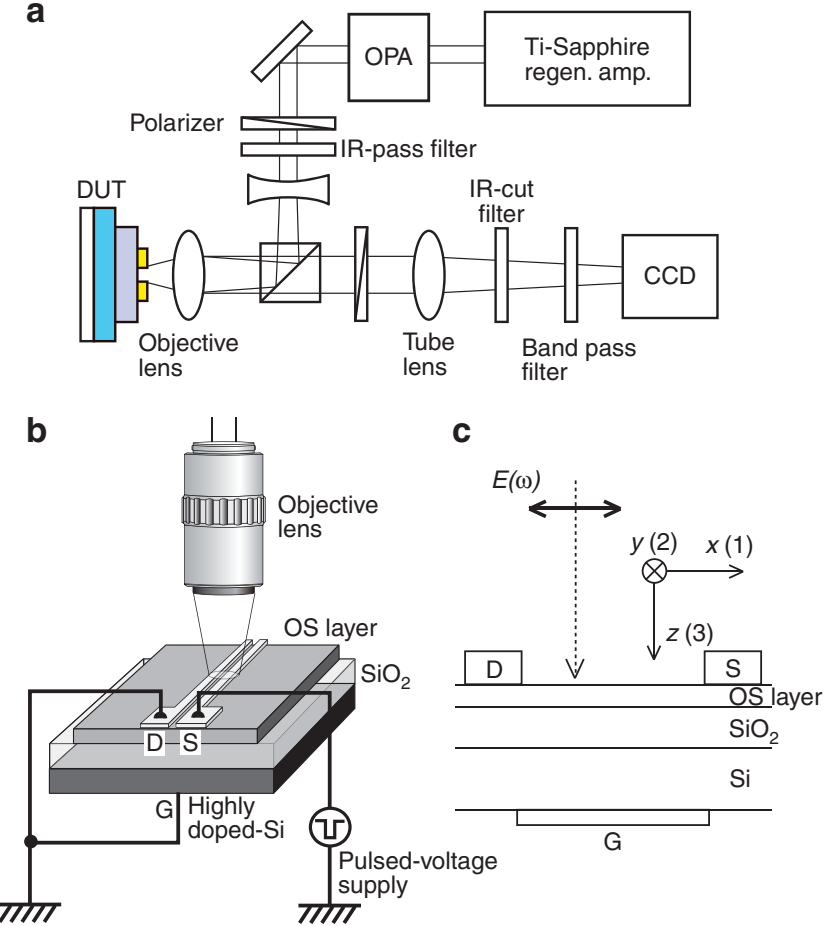

Figure 1 (a) Optical setup for the microscopic SHG measurement. The light source was a femtosecond optical parametric amplifier pumped by a $\mathrm{Ti}$ : Sapphire-regenerative (regen.) amplifier (amp.) system. (b) Device structure and the electrical connection of the TRM-SHG measurement. (c) Optical geometry for the SHG measurement. CCD, charge-coupled device; DUT, device under test; IR, infra-red; OS, organic semiconductor.

polarizations in such films in the presence of the static electric field and the fundamental light are expressed as

$$
\begin{aligned}
P_{1}^{2 \omega}= & \chi_{1111}^{(3)} E_{1}^{0} E_{1}^{\omega} E_{1}^{\omega}+\chi_{1122}^{(3)} E_{1}^{0} E_{2}^{\omega} E_{2}^{\omega}+\chi_{1133}^{(3)} E_{1}^{0} E_{3}^{\omega} E_{3}^{\omega}+2 \chi_{1212}^{(3)} E_{2}^{0} E_{1}^{\omega} E_{2}^{\omega} \\
& +2 \chi_{1313}^{(3)} E_{3}^{0} E_{1}^{\omega} E_{3}^{\omega} \\
P_{2}^{2 \omega}= & 2 \chi_{1212}^{(3)} E_{1}^{0} E_{1}^{\omega} E_{2}^{(\omega)}+\chi_{1122}^{(3)} E_{2}^{0} E_{1}^{\omega} E_{1}^{\omega}+\chi_{1111}^{(3)} E_{2}^{0} E_{2}^{\omega} E_{2}^{\omega}+\chi_{1133}^{(3)} E_{2}^{0} E_{3}^{\omega} E_{3}^{\omega} \\
& +2 \chi_{1313}^{(3)} E_{3}^{0} E_{2}^{\omega} E_{3}^{\omega} \\
P_{3}^{2 \omega}= & 2 \chi_{3113}^{(3)}\left(E_{1}^{0} E_{1}^{\omega} E_{3}^{\omega}+E_{2}^{0} E_{2}^{\omega} E_{3}^{\omega}\right)+\chi_{3311}^{(3)}\left(E_{3}^{0} E_{1}^{\omega} E_{1}^{\omega}+E_{3}^{0} E_{2}^{\omega} E_{2}^{\omega}\right) \\
& +\chi_{3333}^{(3)} E_{3}^{0} E_{3}^{\omega} E_{3}^{\omega}
\end{aligned}
$$

where $E_{1}^{0}$ and $E_{1}^{\omega}$, respectively, represent the static electric field and the electric field of the fundamental light in the $x$-direction. According to Maxwell's equations in media with no birefringence, for example, isotropic media, the $x$-component of the electric field of SHG light $\left(E_{1}^{2 \omega}\right)$ is generated by the $x$-component of the polarization $\left(P_{1}^{2 \omega}\right)$ for normal incidence. The above equation implies that the electric field inside the material along a particular direction is evaluated by selecting the polarization of light. Nevertheless, the third-order nonlinear optical susceptibility of materials should be known for adequate field evaluation.

Because the molecular hyperpolarizability originates from nonlinear interaction between electrons in the molecule and the electric field of light, the nonlinear optical susceptibility is a function of the wavelength. The quantum mechanical perturbation theory allows us to calculate the molecular hyperpolarizability for the EFISHG, $\gamma_{i j k l}^{D C}$, which is easily obtained from the third-order molecular hyperpolarizability a ${ }^{27}$

$$
\begin{aligned}
\gamma_{i j k l}^{D C} & \propto \sum_{m, n, o}\left(\frac{\left\langle g\left|\hat{\mu}_{i}\right| o\right\rangle\left\langle o\left|\hat{\mu}_{j}\right| n\right\rangle\left\langle n\left|\hat{\mu}_{k}\right| m\right\rangle\left\langle m\left|\hat{\mu}_{l}\right| g\right\rangle}{\left(\omega_{o g}-2 \omega\right)\left(\omega_{n g}-2 \omega\right)\left(\omega_{m g}-\omega\right)}\right. \\
+ & \frac{\left\langle g\left|\hat{\mu}_{j}\right| o\right\rangle\left\langle o\left|\hat{\mu}_{i}\right| n\right\rangle\left\langle n\left|\hat{\mu}_{k}\right| m\right\rangle\left\langle m\left|\hat{\mu}_{l}\right| g\right\rangle}{\omega_{o g}\left(\omega_{n g}-2 \omega\right)\left(\omega_{m g}-\omega\right)} \\
+ & \frac{\left\langle g\left|\hat{\mu}_{j}\right| o\right\rangle\left\langle o\left|\hat{\mu}_{k}\right| n\right\rangle\left\langle n\left|\hat{\mu}_{i}\right| m\right\rangle\left\langle m\left|\hat{\mu}_{l}\right| g\right\rangle}{\omega_{o g}\left(\omega_{n g}+2 \omega\right)\left(\omega_{m g}-\omega\right)} \\
+ & \left.\frac{\left\langle g\left|\hat{\mu}_{j}\right| o\right\rangle\left\langle o\left|\hat{\mu}_{k}\right| n\right\rangle\left\langle n\left|\hat{\mu}_{l}\right| m\right\rangle\left\langle m\left|\hat{\mu}_{i}\right| g\right\rangle}{\omega_{o g}\left(\omega_{n g}+2 \omega\right)\left(\omega_{m g}+\omega\right)}\right)
\end{aligned}
$$

Equation (4) becomes the microscopic expression of the molecular hyperpolarizability for third-harmonic generation if we replace the term $\omega_{o g}-2 \omega$ in the denominator by $\omega_{o g}-3 \omega$. Optical transition between the same parity states is forbidden for the electric dipole transition. Namely, a transition dipole moment, $\langle n|\hat{\mu}| m\rangle$, can have a finite value when the parity of the $|n\rangle$ state differs from the parity of the $|m\rangle$ state. From the above equation, we can find the resonant condition for the EFISHG process. The resonance will occur as the wavelength of the SH light approaches the one-photon absorption peak, which implies that the choice of the wavelength is key to effectively observing the EFISHG. Generally, the SHG wavelength should approach the absorption peak to promote resonance enhancement.

\section{EFISHG MEASUREMENTS}

The experimental setup for the EFISHG measurement is shown in Figure 1a. An optical parametric amplifier (OPA) was used as a tunable light source for the EFISHG measurement. The OPA was pumped by a Titanium:Sapphire ultrafast laser system (Coherent, Santa Clara, CA, USA). The repetition rate and pulse width were $1 \mathrm{kHz}$ and $80 \mathrm{fs}$, respectively. The wavelength of the fundamental light was chosen so that the SHG signal could be effectively observed from the devices. For instance, appropriate wavelengths for the pentacene and $\mathrm{C}_{60}$ FETs are $1120 \mathrm{~nm}$ and $1000 \mathrm{~nm}$, respectively. The fundamental light from the OPA was focused onto the OFET channel using a long working distance objective lens (numerical aparture $=0.28$ ). The spot size of the fundamental light was $150 \mu \mathrm{m}$, such that the laser completely irradiated the OFET channel between the drain and the source. The infrared-cut and interference filters were used to eliminate the fundamental and unexpected light from the SHG light generated from the sample. Finally, a highly sensitive charge-coupled device (Andor Technology, Belfast, UK: DU971P-BV) was used to detect the weak SHG light. It should be noted that we can also use the Q-switched YAG laser with a pulse width of few nanoseconds instead of the femtosecond laser system. Use of the femtosecond laser system improves the signal-to-noise ratio of the measurement and reduces the measurement time.

Time-resolved EFISHG measurement is an effective way to probe carrier motion in organic devices. We call this technique time-resolved microscopic SHG (TRM-SHG) measurement. The TRM-SHG measurement is similar to the pump-probe measurement in laser spectroscopy. An electrical stimulus such as pulsed-voltage is applied to the devices instead of a pump beam. Then, we can take a snapshot of the SHG image from the device in the transient state. During the TRMSHG measurement of the OFET, voltage pulses synchronized with laser pulses are applied to the source electrode (see Figure 1b). The delay time, which is the time difference between the rising edge of the voltage pulse and the laser pulse, is strictly controlled to perform the TRM-SHG measurement using a precise delay generator (Stanford 
Research Systems, Sunnyvale, CA, USA: DG645). Temporal resolution is determined by the pulse-width of the laser as well as the rise time of the voltage pulse, that is, the slew rate of the pulse generator and the high-speed amplifier. When we use a nanosecond pulsed laser such as the Q-switched YAG laser, the pulse-width of the laser is almost comparable with the rise time of the voltage pulse, whereas temporal resolution is mainly limited by the electrical equipment when we use the femtosecond laser. For the current setup, the temporal resolution is $\sim 5 \mathrm{~ns}$ when we use the high-speed pulse generator (Avtech Electrosystems, Ottawa, ON, Canada: AV-1011B1-B).

The devices used here for demonstration of the electric field imaging and visualizing carrier motion are top-contact OFETs. We chose $\mathrm{C}_{60}$ and 6,13-Bis(triisopropylsilylethynyl) (TIPS)-pentacene, which are, respectively, known as n- and p-type organic semiconductor materials, for making the FETs. Both molecules have inversion symmetry and intrinsically produce no SHG signal under the dipole approximation. However, as mentioned, these materials are polarized under the electric field and thus produce SHG signals. Heavily-doped Si-wafers coated by a $\mathrm{SiO}_{2}$ layer with a thickness of $500 \mathrm{~nm}$ were used as the substrates. The TIPS-pentacene was deposited on the $\mathrm{Si}$ substrate by dip-coating from a toluene solution with a concentration of $0.5 \mathrm{wt} \%$. The dipping speed was $8.5 \mu \mathrm{m} \mathrm{s}^{-1}$. The $\mathrm{C}_{60}$ was deposited by a vacuum-evaporation method. The process pressure was maintained at $\sim 1 \times 10^{-4} \mathrm{~Pa}$, and the rate of deposition was $3 \mathrm{~nm} \mathrm{~min}{ }^{-1}$. The substrate temperature was not controlled during the evaporation. The thicknesses of the TIPS-pentacene and $\mathrm{C}_{60}$ layers were 60 and $100 \mathrm{~nm}$, respectively. After the deposition of the organic semiconductor materials, 100 -nm-thick gold layers were deposited as the top electrodes by vacuum evaporation. The channel length and width were $30 \mu \mathrm{m}$ and $1.5 \mathrm{~mm}$, respectively, for typical OFET measurements.

\section{EVALUATION OF IN-PLANE ELECTRIC FIELD IN OFETS}

Figure 2a illustrates the SHG image from the channel of the $\mathrm{C}_{60}$ FET under the application of positive voltage to the drain and source electrodes with respect to the gate electrode. It is known that the $\mathrm{C}_{60}$ FET shows n-type FET characteristics even when using an $\mathrm{Au}$ electrode $^{28-30}$. This result indicates that holes are not likely to be injected from the $\mathrm{Au}$ drain and source electrodes into the $\mathrm{C}_{60}$. Although the barrier height estimated by the simple Mott-Schottky relationship is almost comparable for hole and electron injection, the interfacial dipole layer might reduce the barrier for electron injection. The EFISHG profile in Figure $2 \mathrm{~b}$ was obtained by taking a line scan across the channel from the SHG image shown in Figure 2a. The bright SHG signal is visible at the edge of the drain and source electrodes, owing to the edge effect ${ }^{22}$. The strong in-plane electric field formed between the source-drain and the gate electrodes activates SHG at the edge of the electrode. As mentioned in the previous paper $^{21}$, the width of the SHG emission is roughly coincident with the penetration distance of the electric field, owing to the edge effect evaluated by the numerical field calculation. This result also shows that the SHG profile shown in Figure 2a clearly reflects the distribution of the electric field in the OFET channel in the off-state.

Figure $2 \mathrm{c}$ represents the distribution of the electric field obtained from the SHG distribution. In principle, the amplitude of the electric field can be estimated from the SHG intensity using the following equation:

$$
I^{2 \omega} \propto\left|\chi^{(3)} E(0) E(\omega) E(\omega)\right|^{2}
$$

However, such an evaluation is ambiguous because many parameters influence the SHG intensity, such as the intensity of the fundamental
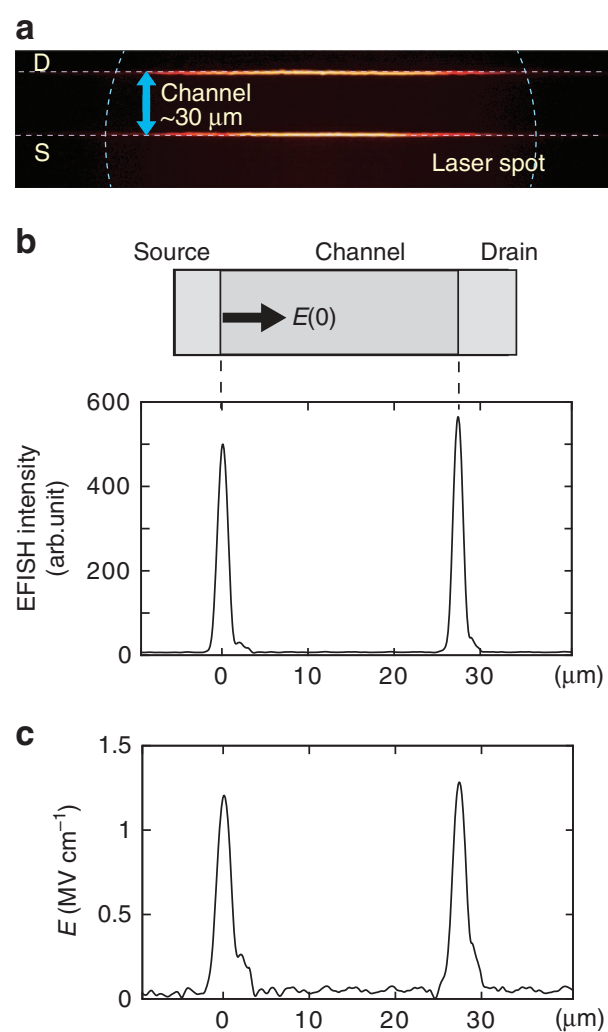

Figure 2 (a) SHG image of the channel in $\mathrm{C}_{60}$ FET under positive pulse application to the source and the drain electrodes. (b) EFISHG profile obtained by taking a line scan across the channel from the SHG image. (c) Electric field distribution obtained from the SHG distribution.

laser and absolute value of the nonlinear optical susceptibility. It is difficult to know the absolute value of all parameters. Therefore, the amplitude of the electric field was evaluated by comparison with the electric field calculated by solving the Laplace equation and the square root of the SHG intensity ${ }^{22}$. The Laplace electric field distribution is uniquely and rigorously determined if the electrode configuration, the dielectric constant and the electric potential on electrodes are provided. It should be noted that the solution of the Laplace equation is used to calibrate the amplitude of the electric field determined by EFISHG, but the field profile is determined directly as $\sqrt{I^{2 \omega}}$. Nevertheless, positive pulses were applied to the drain and source electrodes of the $\mathrm{C}_{60}$ FET to avoid unnecessary carrier injection. Thus, a Laplacian electric field is formed in the OFET corresponding to the off-state. In this review, the distribution of the electric field in the onstate of the FET is not discussed. The SHG intensity distribution in the on-state is completely different from the distribution in the off-state ${ }^{20}$ because of the presence of an additional space charge field formed by the injected carriers.

As mentioned above, the intensity of the EFISHG signal depends on the direction of the static electric field and the nonlinear optical susceptibility tensor of the material. Understanding the relationship between the direction of the static electric field and polarization of the SHG signal is important for probing the electric field as a vectorial quantity. Figure $3 \mathrm{~b}$ and $3 \mathrm{c}$ shows the SHG images around the edge of the square electrode under a parallel and a crossed polarizer, respectively. The static electric fields at the bottom and side edges of the electrode are directed toward the $x$ and $y$ direction, that is, $E_{x}$ and 

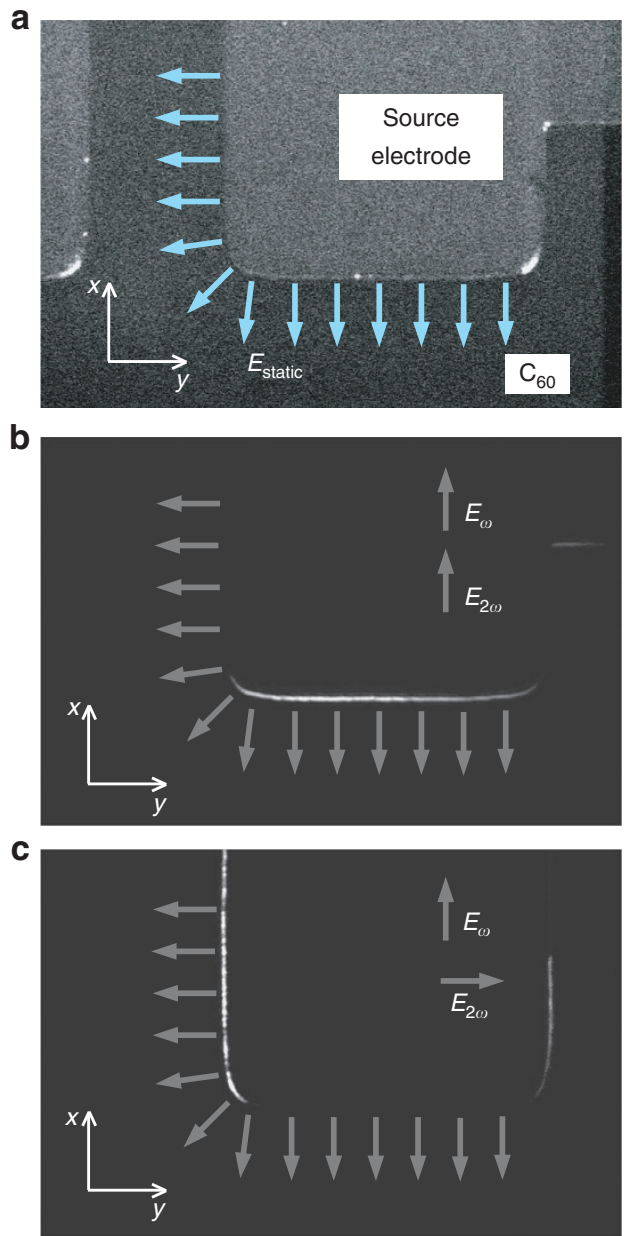

Figure 3 (a) Microscopic image of the edge of the electrode. Arrows schematically show the direction of the electric field. (b) Microscopic SHG image near the edge of the electrode under a parallel polarizer condition. (c) Microscopic SHG image near the edge of the electrode under a crossed polarizer condition (SHG intensity was 10 times magnified).

$E_{y}$, respectively. It should be noted that the field strength is identical $\left(\left|E_{x}\right|=\left|E_{y}\right|\right)$. The direction of the electric field of the fundamental light is changed by changing the angle of the polarizer. Thus, we can choose the directional combination of the static electric field, the electric field of fundamental light and the SHG light by changing the condition of the polarizer.

For a parallel polarization condition, the polarization of the fundamental light is in the $x$-direction $\left(E_{x}(\omega)\right)$ and is coincident with the transmissive axis of the analyzer. Under a parallel polarization condition, we can observe an intense SHG signal at the bottom edge of the electrode, whereas a negligibly small SHG signal is observed at the side edge, as shown in Figure 3b. In contrast, the SHG signal is clearly observed at the side edge under a crossed polarization condition. The SHG intensity from the bottom is $\sim 9$ times larger than the intensity from the side edge. This difference is due to the difference in the nonlinear optical susceptibilities. The SHG signals from the bottom edge and from the side edge originate from different nonlinear polarizations because the direction of the static electric field is different. For the bottom edge, $P_{1}^{2 \omega}=\chi_{1111} E_{1}^{0} E_{1}^{\omega} E_{1}^{\omega}$ is the origin of the SHG signal, whereas $P_{2}^{2 \omega}=\chi_{2211} E_{2}^{0} E_{1}^{\omega} E_{1}^{\omega}$ is the origin of the SHG signal for the side edge. By comparing the SHG intensities from the
Table 1 Independent tensor components of third-order molecular hyperpolarizability for $\mathrm{C}_{60}$ molecule

$\begin{array}{lc}\gamma_{1212}=\gamma_{1221}=\gamma_{1313}=\gamma_{1331}=\gamma_{2121} & \gamma_{1122}=\gamma_{1133}=\gamma_{2211}=\gamma_{2233} \\ =\gamma_{2112}=\gamma_{2323}=\gamma_{2332}=\gamma_{3131}=\gamma_{3113} & =\gamma_{3311}=\gamma_{3322}=0.287 \gamma_{3333} \\ =\gamma_{3232}=\gamma_{3223}=0.356 \gamma_{3333} & \gamma_{1111}=\gamma_{2222}=\gamma_{3333}\end{array}$

bottom and the side edge, the ratio between the two susceptibility components is estimated as $\chi_{2211} / \chi_{1111}=0.29 \sim 0.38$.

As mentioned above, the nonlinear optical susceptibilities are related to the ensemble average of the molecular hyperpolarizabilities. Thus, the third-order molecular hyperpolarizability tensor is evaluated on the basis of quantum chemical calculation, if the information on molecular orientation in the aggregate is available. Here, we show the theoretical estimation of the nonlinear optical susceptibilities to show the validity of the experimental results, that is, the ratio between two susceptibility components. Before the calculation of the molecular hyperpolarizability, the molecular structure of $\mathrm{C}_{60}$ in the ground state was optimized based on a semi-empirical calculation using the AM1 Hamiltonian. Then, the third-order molecular hyperpolarizability for the optimized molecular structure was evaluated. All the calculations are performed by using the MOPAC program package. Table 1 summarizes the relationships among the non-zero components of the third-order molecular hyperpolarizability tensor of an isolated $\mathrm{C}_{60}$ molecule for the EFISHG process. These non-zero components can be expressed using the largest component, $\gamma_{3333}$. Note that $\gamma_{3333}$ is estimated as $2.68 \times 10^{-35}$ esu. This point indicates that the number of independent components is reduced to one, $\gamma_{3333}$, reflecting the high symmetry (icosahedral) of the $\mathrm{C}_{60}$ molecule. After lengthy average calculations, all $\chi_{i j k l}^{(3)}$ components can be expressed using the molecular hyperpolarizability as

$$
\begin{aligned}
\chi_{1111} & =\chi_{2222}=\chi_{3333}=k \gamma_{3333} \\
\chi_{1122} & =\chi_{1133}=\chi_{2211}=\chi_{2233}=\chi_{3311}=\chi_{3322}=0.2827 k \gamma_{3333} \\
\chi_{1212} & =\chi_{1221}=\chi_{1313}=\chi_{1331}=\chi_{2112}=\chi_{2121}=\chi_{2323} \\
& =\chi_{2332}=\chi_{3113}=\chi_{3131}=\chi_{3223}=\chi_{3232}=0.3562 k \gamma_{3333},
\end{aligned}
$$

where $k$ is a proportional constant. Then, the ratio between the susceptibility components is estimated as $\chi_{2211} / \chi_{1111}=0.287$, which is in agreement with the experimental value. The static electric field whose direction is coincident with the polarization of the light is selectively probed.

\section{VISUALIZATION OF CARRIER MOTION IN OFETS}

The electric field discussed above corresponds to the Laplacian field. The Gauss law in electromagnetic theory tells us that electric flux diverges from the charged particles, which implies that the distribution of the electric field in the on-state of the FET may change from the Laplacian field formed in the off-state because of the contribution from the injected carriers. Thus, by analyzing the distribution of the electric field in the on-state, we can also evaluate the carrier distribution in the device. Here, we demonstrate that the transient carrier transport phenomena can be visualized by the EFISHG measurements and time-resolved techniques. In particular, we discuss the anisotropic character of the carrier transport in single-crystal grains of organic semiconductor on the basis of the EFISHG measurement. Analysis of the anisotropic carrier transport is important for the complete understanding of the transport phenomena in the devices, as well as the electric field evaluation. To demonstrate the visualization of the anisotropic carrier 

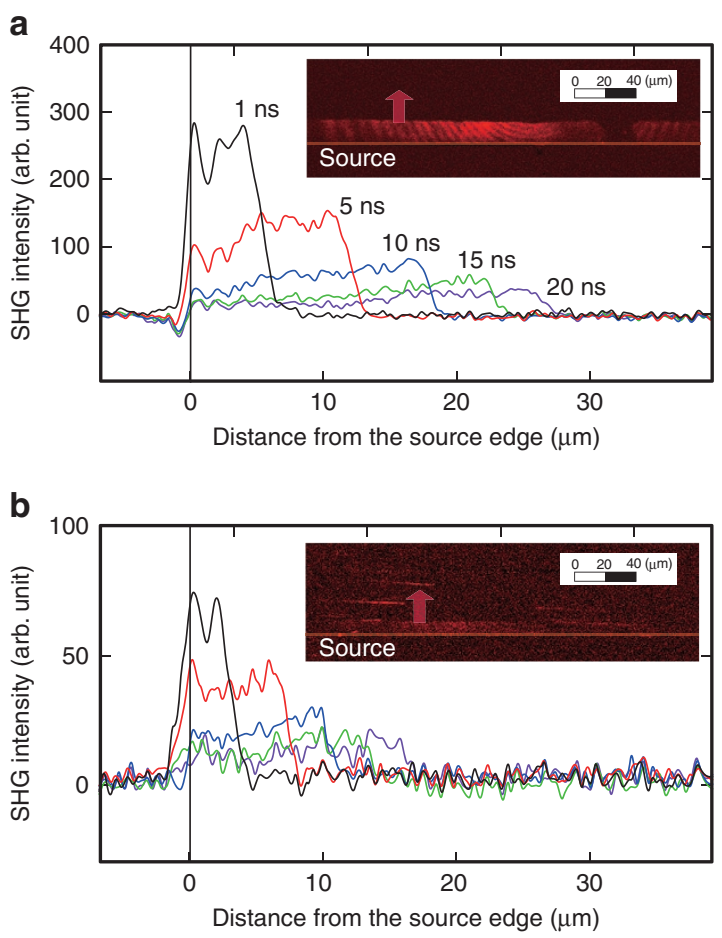

Figure 4 Transient SHG intensity distribution along the FET channel with different delay times. (a) Device in which the dipping direction is parallel to the source-drain direction. (b) Device in which the dipping direction is perpendicular to the source-drain direction. Insets are the actual SHG images from the OFET channel.

transport, we used the FET with TIPS-pentacene. TIPS-pentacene is a derivative of pentacene and is soluble in many solvents ${ }^{31,32}$. When TIPS-pentacene is deposited onto a substrate by the dip-coating method, large grains with a narrow striped shape are spontaneously obtained.

The transient changes in the SHG distribution along the channel of TIPS-pentacene FET are shown in Figure $4 \mathrm{a}$ and $4 \mathrm{~b}$. The difference between Figure $4 \mathrm{a}$ and $4 \mathrm{~b}$ is the dipping direction with respect to the current flow direction. In Figure $4 \mathrm{a}$, the dipping direction is parallel to the source-drain direction, whereas the dipping direction is perpendicular to the source-drain direction in Figure 4b. Hereafter, the former and latter devices are denoted as parallel and perpendicular FETs, respectively. The actual SHG images are displayed in the inset of Figure 4 as examples. These images were captured by applying positive pulses to the source electrode so that holes were injected from the electrode. The amplitude of the voltage pulses was $100 \mathrm{~V}$, and the laser pulses were impinged after $15 \mathrm{~ns}$ of the voltage pulses. The SHG intensity distributions were obtained by taking a line scan of the SHG images along the source-drain direction ${ }^{21}$. As shown in these figures, the front of the SHG emission band moves from the source to the drain electrode. This result indicates the migration of the injected holes in the channel of OFET. The SHG intensity distribution is quite sensitive to the trap density of the channe ${ }^{33}$. The homogeneous SHG distribution observed in Figure 4 during the transient carrier transport indicates a low trap density in a single crystalline grain of TIPSpentacene.

Figure 4 clearly shows a large carrier velocity in the parallel FET compared with the perpendicular one. As reported previously, the

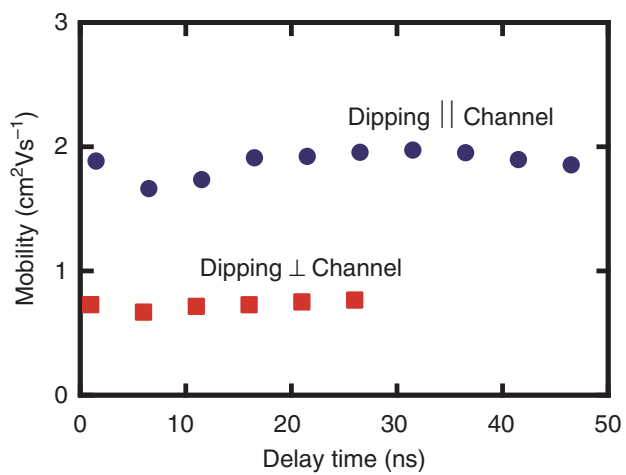

Figure 5 Transient carrier mobility of the samples with different dipping directions calculated by using Equation (7) at different delay times.

transport distance of the carrier is proportional to the square root of time $e^{34,35}$. Thus, we can calculate the carrier mobility using a simple formula

$$
\mu=\frac{x^{2}}{2 V_{s}} t
$$

by assuming a uniform electric field $V_{s} / x$ in the channel region, where $x$ is the length between the edge of source electrode and the injected carrier propagation front. In this equation, $V_{s}$ and $t$ represent the source voltage and the delay time, respectively. The injected carrier propagation front corresponds to the front position of the SHG emission. The obtained SHG distribution supports the assumption of the constant electric field distribution. Figure 5 shows the plot of the transient carrier mobility of the samples with different dipping directions, calculated by using Equation (7) at different delay times. The transient carrier mobilities of the parallel and perpendicular FETs are estimated as $\sim 1.8$ and $0.6 \mathrm{~cm}^{2} \mathrm{~V}^{-1} \mathrm{~s}^{-1}$, respectively, indicating a mobility anisotropy $\mu_{/ /} / \mu_{\perp}$ of $\sim 3.0$. It should be noted that $\mathrm{V}$. Gulbinas has successfully revealed the ultrafast dynamics of carrier mobility in a $\pi$-conjugated polymer on the basis of pump-probe SHG measurement ${ }^{36}$. The results successfully showed the time-resolved mobility changing from $\sim 0.1 \mathrm{~cm}^{2} \mathrm{~V}^{-1} \mathrm{~s}^{-1}$ at 1 ps to its stationary value of $\sim 10^{-6} \mathrm{~cm}^{2} \mathrm{~V}^{-1} \mathrm{~s}^{-1}$, which indicates that the mobility in a short timeframe $(<1 \mathrm{~ns})$ is determined by electrons but that the mobility at a sub-microsecond timeframe is attributed to holes.

Recently, we have proposed a novel method to observe anisotropic carrier transport by TRM-SHG measurement using circular-shaped electrode ${ }^{37}$. Because the circular-shaped electrode can inject carriers uniformly around the electrode, angle-dependent mobility is visualized directly and simultaneously. Figure $6 \mathrm{~b}$ shows the TRM-SHG images of a TIPS-pentacene single crystalline grain covered with a circularshaped electrode at a delay time of 20 ns. Interestingly, the carriers spread from the electrode, but the SHG distribution is not a complete circle, indicating that the velocity of the carrier strongly depends on the flow direction. In other words, the elliptical shape of the SHG image directly represents the anisotropy of the carrier mobility. When we performed the same TRM-SHG measurement for the vacuumevaporated pentacene device, mobility anisotropy was not observed, indicating the macroscopic in-plane isotropy of the film. It should be noted that the drain electrode is not necessary for this measurement, as the injected carriers are transported along the channel owing to the space charge field formed by the injected carriers itself $f^{37}$, as modeled by charging process of a RC ladder circuit ${ }^{38,39}$. 
a

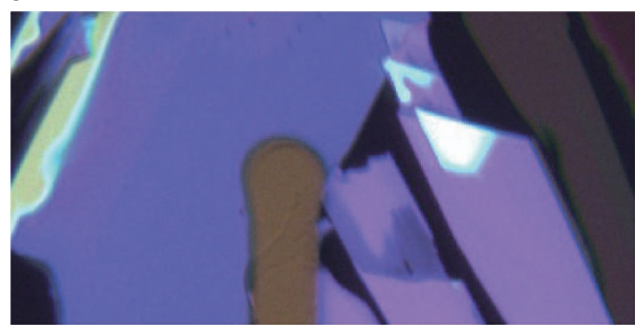

b

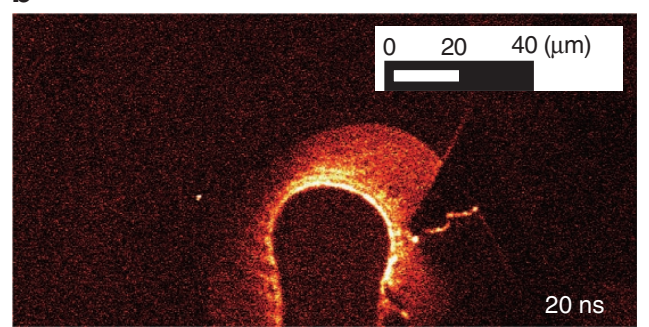

Figure 6 (a) Polarized microscopic image of the circular Au electrode. (b) TRM-SHG images from TIPS-pentacene single crystalline grain covered with a circular-shaped electrode at a delay time of $20 \mathrm{~ns}$.

The SHG intensity on the $\mu_{\max }$-axis is strong compared with the intensity on the $\mu_{\mathrm{min}}$-axis, which is due to the tensor character of the nonlinear optical susceptibility $\chi^{(3)}$ of the TIPS-pentacene single crystal and the direction of the electric field vector of light. The $\chi^{(3)}$ components strongly depend on the delocalization of the $\pi$-electrons in the molecular system. The presence of anisotropic mobility represents the anisotropic inter-molecular interaction in the crystal. The transfer integral along the $\mu_{\max }$-axis becomes dominant, and the delocalization of the $\pi$-electrons along this direction is extended. In this sense, it is reasonable to consider that the $\chi^{(3)}$ components become dominant along the $\mu_{\max }$-axis.

Even within single crystalline grains, carrier transport is sometimes governed by defects and invisible grain boundaries. However, the carrier behavior at such defects and grain boundaries cannot be evaluated from a simple I-V measurement because only the averaged I-V characteristics of the whole channel are obtained. However, the TRM-SHG measurement directly visualizes the intra-grain and intergrain carrier transport. In other words, SHG microscopy provides local information. For example, Figure 7a shows the TRM-SHG image of the TIPS-pentacene FET at a delay time of $70 \mathrm{~ns}$. As shown in the figure, a bright line was observed in the channel (see inside dashed circle). The generation of the strong SHG signal implies a strong electric field. The presence of a transport barrier at this point induces accumulation of the charges, and these charges are the origin of the strong electric field. Although the origin of this transport barrier is not revealed at present, a similar pattern was observed more faintly in the polarized microscope image shown in Figure 7b. Presumably, a small defect in the grain at that point disturbed the carrier transport. Nevertheless, the effect of the grain boundary on the carrier transport is visualized by the TRM-SHG measurement.

Vibrational spectroscopy is a valuable tool for investigating carrier transport at a molecular level because of its sensitivity to the molecular structure and orientation. Gracias and co-workers have performed SFG measurement during the operation of the OFET ${ }^{13,14}$. SFG is also a surface and interface selective vibrational spectroscopy and is suitable for application to OFET because a very thin channel is formed near a

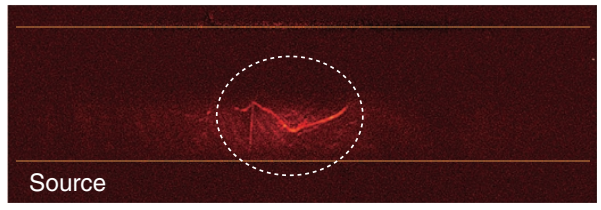

b

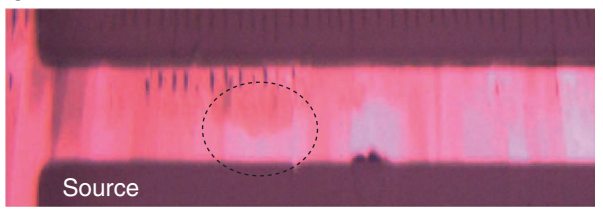

Figure 7 (a) Bright line observed in the SHG image from the channel of TIPS-pentacene FET (see inside a dashed circle). (b) Polarized microscope image around the same area.

the gate dielectric. They successfully observed the change in the SFG spectra upon applying a gate voltage. With increasing negative gate voltage, the increase in the ratio of the $\mathrm{CH}_{2}$-ss peak to the $\mathrm{CH}_{3}$-ss peak intensity was observed, indicating a structural change in the alkyl chain due to the presence of the delocalized charge.

\section{SOME APPLICATIONS OF THE EFISHG MEASUREMENT TO OTHER DEVICES}

Carrier behavior in various types of organic devices, such as OLEDs and OSCs, is also directly probed by the EFISHG measurement. However, attention must be paid to the difference in device structure between OLED (OSC) and OFET for the optical setup. As mentioned above, the in-plane electric field governs the carrier transport in the OFET channel. In contrast, the out-of-plane (longitudinal) field is important for sandwiched devices such as OLEDs and OSCs because the current flows across the film. According to Equation (3), under the assumption of $C_{\infty v}$ symmetry of the film, the $z$ component of the electric field of light $\left(E_{3}^{\omega}\right)$ is required to detect the longitudinal static electric field $\left(E_{3}^{0}\right)$. Thus, we must use the oblique incidence of p-polarized light to probe the carrier motion and electric field in these sandwiched devices. Here, we show a brief summary of the EFI SHG measurement for OLEDs ${ }^{40-44}$ and OSCs ${ }^{45-50}$.

\section{APPLICATION TO OLED}

In OLED devices, electrons and holes are injected from electrodes with appropriate work-functions into the electron and hole transport layers, respectively. Figure 8a shows the experimental configuration of the SHG measurement for an OLED. The p-polarized fundamental light is incident to the device, and p-polarized SHG light is detected. The OLED is operated by applying a pulsed voltage. After applying the pulsed voltage to the device, some of the injected carriers accumulate at the interface between the hole and electron transport layers, owing to the Maxwell-Wagner effect ${ }^{51}$. They then recombine, leading to electroluminescence. Thus, probing carrier behavior in the constituent layers of the OLED is an effective way to analyze the device operation. To observe the carrier behavior in each layer, we can take advantage of the spectroscopic selectivity of optical measurement. Figure $8 \mathrm{~b}$ schematically shows the SHG spectrum of a double-layered structure. Each organic material has its own intrinsic optical spectrum. This property allows us to probe the electric field in each layer of a double-layer structure by selecting the proper fundamental wavelength. Figure $8 \mathrm{c}$ schematically represents the transient SHG response and the 

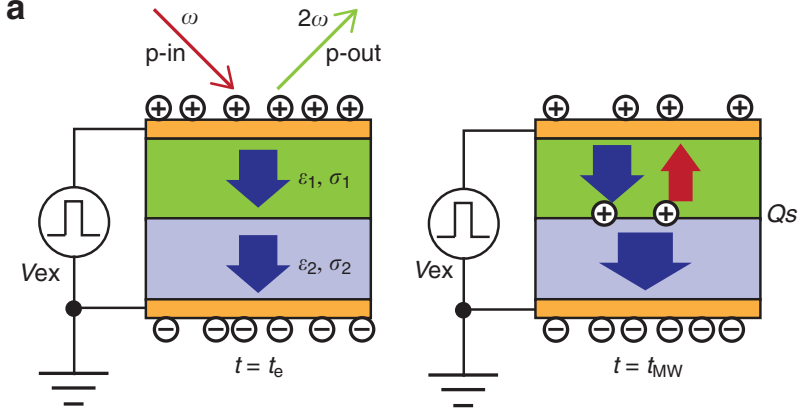

b

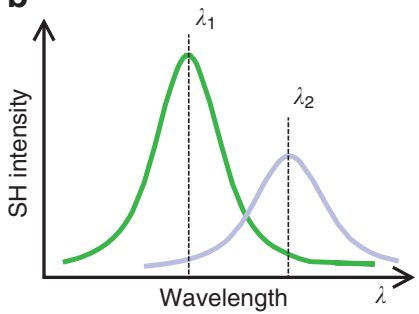

c

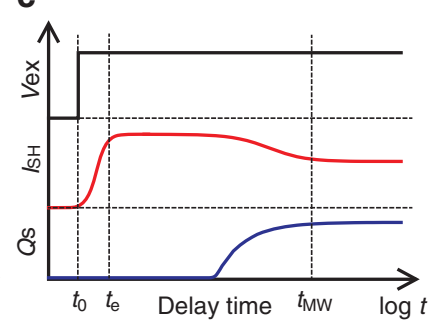

Figure 8 (a) Experimental configuration of the SHG measurement for OLED. (b) Schematic image of the SHG spectrum of double-layered structure. (c) Transient SHG response and the accumulated carriers with respect to the voltage pulse. $\mathrm{SH}$, second-harmonic.

accumulated carriers with respect to the delay time. The SHG intensity increases and saturates at $t=t_{e}$, which indicates the completion of charging of the electrodes. The SHG intensity remains nearly constant during the carrier transport process in the transport layers and afterward begins to decrease, then saturates again at the MaxwellWagner time constant, $t=t_{\mathrm{MW}}$. This result is due to the MaxwellWagner charging at the interface. As shown in Figure 8a, holes accumulate at the interface, and the electric field generated from the accumulated holes partially cancels out the external electric field, which weakens the SHG intensity. Thus, EFISHG enables us to directly observe the accumulation of injected carriers at the interface.

We first applied the time-resolved EFISHG technique to an OLED with an $\mathrm{IZO} / \alpha-\mathrm{NPD} / \mathrm{Alq}_{3} / \mathrm{LiF} / \mathrm{Al}$ structure ${ }^{40}$. To selectively probe the SHG signal from the $\alpha$-NPD layer, wavelength of the fundamental light was set to $820 \mathrm{~nm}$. Immediately after applying the forward-bias voltage, SHG intensity from the $\alpha$-NPD layer increases with a specific response time. This response time is in good agreement with the resistor-capacitor circuit time constant of the series capacitance of $\alpha-\mathrm{NPD}$ and $\mathrm{Alq}_{3}$ layers and the lead resistance of an IZO electrode. The decrease in the SHG intensity clearly indicates that the positive charges are accumulated at the $\alpha-\mathrm{NPD} / \mathrm{Alq}_{3}$ interface, and it is accompanied by luminescence due to the charge recombination. By analyzing the SHG intensity, the amplitude of the electric field in the $\alpha$-NPD layer and the amount of accumulated charge can be quantitatively obtained.

\section{APPLICATION TO OSC}

OSCs are promising devices for next-generation power sources, and many studies have been conducted to increase their conversion efficiency ${ }^{52}$. As in OLED devices, the fundamental behavior of carriers that leads to the photovoltaic effect should be considered when analyzing the device operation. Assuming a bilayer-type OSC device consisting of $\mathrm{n}$ - and p-type molecules, excitons created in each layer under photo-illumination diffuse to the $\mathrm{p} / \mathrm{n}$ interface, followed by

dissociation into free electrons and holes. Then, the generated holes and electrons are transported across the $\mathrm{p}$ - and n-layers to the electrodes, respectively. Consequently, photocurrent flows through the external circuit. We attempted to observe the carrier accumulation and decay processes at the $\mathrm{p} / \mathrm{n}$ interface of an $\mathrm{IZO} /$ pentacene $/ \mathrm{C}_{60} / \mathrm{Al}$ OSC by EFISHG measurement ${ }^{47}$. A fundamental wavelength of $1000 \mathrm{~nm}$ was used to selectively observe the SHG signal from the $\mathrm{C}_{60}$ layer.

In the short-circuit condition, the SHG intensity initially increased under red-light illumination and finally saturated. The saturated SHG signal decreased and returned to its intrinsic intensity after the illumination was stopped. These results clearly indicate that charges were generated at the interface between pentacene and $\mathrm{C}_{60}$ under illumination. The photo-voltage generated at the pentacene $/ \mathrm{C}_{60}$ interface was estimated by analyzing the SHG intensity, and it was $V_{s}=0.16 \mathrm{~V}$ with respect to the $\mathrm{Al}$ electrode. This estimation is critical in discussing the open-circuit voltage $V_{o c}$. $V_{o c}$ is governed by the energy difference between the lowest unoccupied molecular orbital in the acceptor and the highest occupied molecular orbital in the donor, which is called the built-in potential ${ }^{53}$. However, it varies due to the accumulated charges at the interface between donor and acceptor. According to the $\mathrm{I}-\mathrm{V}$ characteristics, the open-circuit voltage was measured as $0.24 \mathrm{~V}$, which reflects the effect of the accumulate charge. It is noteworthy that the EFISHG is also useful for studying carrier processes in blended-type OSCs, that is, bulk heterojunction OSCs ${ }^{51}$. We successfully observed the SHG signal from individual components in bulk heterojunction OSCs by selecting appropriate wavelengths of fundamental light. These results reveal the presence of different paths for electrons and holes in bulk heterojunction OSCs.

The above-mentioned time-resolved SHG measurements show the carrier transport and accumulation processes in OLEDs and OSCs with a temporal range of few $\mu$ s. Time-resolved SHG can also be used to demonstrate the exciton dynamics in the OSC with a subpicosecond temporal range. Zhu and co-workers have examined the dynamic nature of the charge-transfer (CT) excitons at the donor/ acceptor interface based on ultrafast time-dependent pump-probe measurement by combining time-resolved SHG with two-photon photoemission $^{12}$. They showed the direct and indirect formation of $\mathrm{CT}$ excitons at the $\mathrm{CuPc} /$ fullerene interface by changing the excitation wavelength. Here, CT excitons are formed directly by optical excitation, and indirect CT excitons are created by the dissociation of molecular excitons. The time-constant of the establishment of interfacial electric fields was different between direct and indirect CT excitons because the formation of the indirect $\mathrm{CT}$ excitons requires the dissociation process.

\section{APPLICATION TO INORGANIC POWER DEVICES}

This technique can be applied not only to organic devices but also to inorganic devices. Here, we introduce the application of the EFISHG technique to amorphous silicon (a-Si) solar cells ${ }^{54}$ and AlGaN/GaN high electron mobility transistors ${ }^{55}$. For the a-Si solar cells, we investigated the SHG signal generated from a-Si:H solar cells and a-Si/microcrystalline silicon ( $\mu \mathrm{c}-\mathrm{Si})$ double-junction solar cells. The SHG signal originating from the intrinsic layer strongly depended on the external voltage $\left(V_{\text {ex }}\right)$, whereas the SHG signal generated from the a-Si interface was not dependent on the external voltage. However, in double-junction cells, the interfacial $\mathrm{p}-\mathrm{n}$ junction of a-Si and $\mu \mathrm{c}-\mathrm{Si}$ may influence the SHG. These results imply that the EFISHG measurement may provide a novel nondestructive technique for studying inorganic semiconducting devices. Gallium nitride $(\mathrm{GaN})$ is a potential candidate material for electronic power devices owing to its 
distinguishing properties, such as its high breakdown voltage. However, the drain current in the on-state of a Schottky gate AlGaN/GaN high electron mobility transistors decreases after application of the drain voltage in the off-state. This reduction of the current is known as current collapse caused by the trapped charges. To understand the mechanism of the current collapse, two-dimensional current collapse imaging was conducted using EFISHG measurements. On the basis of the time-resolved measurements, this technique successfully visualized the trapped carrier within a short time after the transition from the off- to on-states, compared with probe microscope-based measurement such as Kelvin force microscopy.

\section{CONCLUSIONS}

In this review, we have briefly summarized our technique developed to visualize electric fields and carrier motions in organic materials and devices based on microscopic EFISHG measurements. By applying this technique, the in-plane electric field distribution and transient carrier motion in the OFET were directly observed with high spatial and temporal resolution. The temporal and the spatial resolutions of this system are $5 \mathrm{~ns}$ and $0.5 \mu \mathrm{m}$, respectively. The results obtained by the EFISHG measurements will also contribute to the further development of organic device physics.

\section{CONFLICT OF INTEREST}

The authors declare no conflict of interest.

\section{ACKNOWLEDGEMENTS}

Financial support by the Grants-in-Aid for Scientific Research (no. 22226007, 21686029,24360118 , and 15H03971) from the Japan Society for the Promotion of Science is greatly appreciated.

1 Zhou LS, Wanga A, Wu SC, Sun J, Park S et al. All-organic active matrix flexible display. Appl Phys Lett 2006; 88: 083502.

2 Lodha A, Singh R. Prospects of manufacturing organic semiconductor-based integrated circuits. IEEE Trans Semi Manu 2001; 14: 281-296.

3 Nonnenmacher M, O'Boyle MP, Wickramasinghe HK. Kelvin probe force microscopy. Appl Phys Lett 1991; 58: 2921-2923.

4 Vatel 0 , Tanimoto M. Kelvin probe force microscopy for potential distribution measurement of semiconductor devices. J Appl Phys 1995; 77: 2358-2362.

5 Kikukawa A, Hosaka S, Imura R. Silicon pn junction imaging and characterizations using sensitivity enhanced Kelvin probe force microscopy. Appl Phys Lett 1995; 66 3510-3512.

6 Mizutani T, Arakawa M, Kishimoto S. Two-dimensional potential profile measurement of GaAs HEMT's by Kelvin probe force microscopy. IEEE Electr Dev Lett 1997; 18 423-425.

7 Bürgi L, Sirringhaus H, Friend RH. Noncontact potentiometry of polymer field-effect transistors. App/ Phys Lett 2002; 80: 2913-2915.

8 Ohashi N, Tomii H, Matsubara R, Sakai M, Kudo K et al. Conductivity fluctuation within a crystalline domain and its origin in pentacene thin-film transistors. App/ Phys Lett 2007; 91: 162105.

9 Beljonne D, Cornil J, Sirringhaus H, Brown PJ, Shkunov M et al. Optical signature of delocalized polarons in conjugated polymers. Adv Funct Mater 2001; 11: 229-234.

10 Itoh E, Terashima K, Nagai H, Miyairi K. Evaluation of poly(3-hexylthiophene)/polymeric insulator interface by charge modulation spectroscopy technique. Thin Solid Films 2009; 518: 810-813.

11 Manaka T, Kawashima S, Iwamoto M. Charge modulated reflectance topography for probing in-plane carrier distribution in pentacene field-effect transistors. App/ Phys Lett 2010; 97: 113302.

12 Jailaubekov AE, Willard AP, Tritsch JR, Chan WL, Sai N et al. Hot charge-transfer excitons set the time limit for charge separation at donor/acceptor interfaces in organic photovoltaics. Nat Mater 2013; 12: 66-73.

13 Ye HK, Abu-Akeel A, Huang J, Katz HE, Gracias DH. Probing organic field effect transistors in situ during operation using SFG. J Am Chem Soc 2006; 128: 6528-6529.

14 Ye HK, Huang J, Park JR, Katz HE, Gracias DH. Correlations between SFG spectra and electrical properties of organic field effect transistors. J Phys Chem B 2007; 111: 13250-13255.

15 Lampert MA, Mark P. Current Injection in Solids. New York: Academic Press; 1970.
16 Morris JD, Atallah TL, Lombardo CJ, Park H, Dodabalapur A et al. Mapping electric field distributions in biased organic bulk heterojunctions under illumination by nonlinear optical microscopy. Appl Phys Lett 2013; 102: 033301.

17 Sciascia C, Celebrano M, Binda M, Natali D, Lanzani G et al. Electric field and charge distribution imaging with sub-micron resolution in an organic thin-film transistor. Org Electron 2012; 13: 66-70.

18 Manaka T, Lim E, Tamura R, Iwamoto M. Direct imaging of carrier motion in organic transistors by optical second-harmonic generation. Nat Photon 2007; 1: 581-584.

19 Iwamoto M, Manaka T, Taguchi D. Probing and modeling of carrier motion in organic devices by electric-field-induced optical second-harmonic generation. Jpn J Appl Phys 2014; 53: 100101.

20 Manaka T, Lim E, Tamura R, Yamada D, Iwamoto M. Probing of the electric field distribution in organic field effect transistor channel by microscopic second-harmonic generation. Appl Phys Lett 2006; 89: 072113.

21 Manaka T, Nakao M, Yamada D, Lim E, Iwamoto M. Optical second harmonic generation imaging for visualizing in-plane electric field distribution. Opt Express 2006; 15: 15964-15971.

22 Yamada D, Manaka T, Lim E, Tamura R, Weis M et al. Injected carrier distribution in a pentacene field effect transistor probed using optical second harmonic generation. J App/ Phys 2008; 104: 074502.

23 Levine BF, Bethea CG. Second and third order hyperpolarizabilities of organic molecules. J Chem Phys 1975; 63: 2666-2682.

24 Bosshard C, Knöpfle G, Prêtre P, Günter P. Second-order polarizabilities of nitropyridine derivatives determined with electric-field-induced second-harmonic generation and a solvatochromic method: A comparative study. J Appl Phys 1992; 71: 1594-1605.

25 Rose ME. Elementary Theory of Angular Momentum. New York: Wiley; 1957.

26 Popov SV, Svirko YP, Zheludev NI. Susceptibility Tensors for Nonlinear Optics. Bristol: Institute of Physics Publishing; 1995.

27 Boyd RW. Nonlinear Optics. Amsterdam: Academic Press; 2003.

28 Kitamura M, Arakawa Y. Pentacene-based organic field-effect transistors. J Phys Condens Matter 2008; 20: 184011.

29 Cai XY, Yu JS, Zhou JL, Yu XG, Jiang YD. Mobility improvement in $\mathrm{C}_{60}$-based field-effect transistors using LiF/Ag source/drain electrodes. Jpn J App/ Phys 2011; 50: 124203.

30 Kobayashi S, Takenobu T, Mori S, Fujiwara A, Iwasa Y. Fabrication and characterization of $\mathrm{C}_{60}$ thin-film transistors with high field-effect mobility. Appl Phys Lett 2003; 82 4581-4583.

31 Ostroverkhova O, Cooke DG, Hegmann FA, Tykwinski RR, Parkin SR et al. Anisotropy of transient photoconductivity in functionalized pentacene single crystals. Appl Phys Lett 2006; 89: 192113.

32 Sele CW, Kjellander BKC, Niesen B, Thornton MJ, Putten J et al. Controlled deposition of highly ordered soluble Acene thin films: effect of morphology and crystal orientation on transistor performance. Adv Mater 2006; 21: 4926-4931.

33 Manaka T, Liu F, Weis M, Iwamoto M. Influence of traps on transient electric field and mobility evaluation in organic field-effect transistors. J Appl Phys 2010; 107: 043712.

34 Manaka T, Liu F, Weis M, Iwamoto M. Diffusion like electric-field migration in the channel of organic field-effect transistors. Phys Rev B 2008; 78: 121302

35 Manaka T, Liu F, Weis M, Iwamoto M. Studying transient carrier behaviors in pentacene field effect transistors using visualized electric field migration. J Phys Chem C 2009; 113: 10279-10284.

36 Devizis A, Serbenta A, Meerholz K, Hertel D, Gulbinas V. Ultrafast dynamics of carrier mobility in a conjugated polymer probed at molecular and microscopic length scales. Phys Rev Lett 2009; 103: 027404.

37 Manaka T, Matsubara K, Abe K, Iwamoto M. Direct observation of anisotropic carrier transport in organic semiconductor by time-resolved microscopic optical secondharmonic imaging. Appl Phys Express 2013; 6: 101601.

38 Weis M, Manaka T, Iwamoto M. Origin of electric field distribution in organic field-effect transistor: Experiment and analysis. J Appl Phys 2009; 105: 024505.

39 Weis M, Lin J, Taguchi D, Manaka T, Iwamoto M. The charge transport in organic fieldeffect transistor as an interface charge propagation: The maxwell-wagner effect model and transmission line approximation. Jpn J App/ Phys 2010; 49: 071603.

40 Taguchi D, Inoue S, Zhang L, Li J, Weis M et al. Analysis of organic light-emitting diode as a maxwell-wagner effect element by time-resolved optical second harmonic generation measurement. J Phys Chem Lett 2010; 1: 803-807.

41 Taguchi D, Zhang L, Li J, Weis M, Manaka T et al. Direct probing of carrier behavior in electroluminescence indium-zinc-oxide/ $N, \quad N$-Di-[(1-naphthyl)- $N, \quad N^{\prime}$-diphenyl]-(1, 1'-biphenyl)-4, 4'-diamine/Tris(8-hydroxy-quinolinato)aluminum(III)/LiF/Al diode by time-resolved optical second-harmonic generation. Jpn J Appl Phys 2011; 50: 04DK08.

42 Sadakata A, Taguchi D, Yamamoto T, Fukuzawa M, Manaka T et al. Analyzing two electroluminescence modes of indium tin oxide/ $\alpha-N P D / A l q 3 / A$ l diodes by using large alternating current square voltages. J Appl Phys 2011; 110: 103707.

43 Sadakata A, Osada K, Taguchi D, Yamamoto T, Fukuzawa M et al. Probing interfacial charge accumulation in ITO/ $\alpha-\mathrm{NPD} / \mathrm{Alq} 3 / \mathrm{Al}$ diodes under two electroluminescence operational modes by electric-field induced optical second-harmonic generation. J Appl Phys 2012; 112: 083723.

44 Sadakata A, Oda Y, Taguchi D, Fukuzawa M, Manaka T et al. Study of hole-injection assisted layer in double-layer organic light-emitting diodes by electric-field-induced optical second-harmonic generation measurement. Jpn J Appl Phys 2013; 52: 05DC03. 
45 Taguchi D, Shino T, Chen XY, Zhang L, Li J et al. Analyzing carrier lifetime of doublelayer organic solar cells by using optical electric-field-induced second-harmonic generation measurement. Appl Phys Lett 2011; 98: 133507.

46 Taguchi D, Shino T, Chen XY, Zhang L, Li J et al. Analyzing photovoltaic effect of double-layer organic solar cells as a Maxwell-Wagner effect system by optical electricfield-induced second-harmonic generation measurement. J App/ Phys 2011; 110: 103717.

47 Taguchi D, Shino T, Zhang L, Li J, Weis M et al. Direct probing of photovoltaic effect generated in double-layer organic solar cell by electric-field-induced optical secondharmonic generation. App/ Phys Express 2011; 4: 021602.

48 Chen XY, Taguchi D, Lee K, Manaka T, Iwamoto M. Analyzing interfacial carrier charging in pentacene/ $C_{60}$ double-layer organic solar cells by optical electric field induced second-harmonic generation measurement. Chem Phys Lett 2011; 511: 491-495.

49 Chen XY, Taguchi D, Weis M, Manaka T, Iwamoto M. Analyzing photo induced internal electric field in pentacene/ $\mathrm{C}_{60}$ double-layer organic solar cells under various external voltages by electric-field-induced optical second harmonic generation measurement. Jpn J Appl Phys 2012; 51: 041605.

50 Chen XY, Taguchi D, Lee K, Manaka T, Iwamoto M. Analysis of interfacial charging process in pentacene/ $\mathrm{C}_{60} /$ bathocuproine triple-layer organic solar cells using a maxwellwagner model. Jpn J Appl Phys 2013; 52: 04CR05.
51 Tamura R, Lim E, Manaka T, Iwamoto M. Analysis of pentacene field effect transistor as a Maxwell-Wagner effect element. J Appl Phys 2006; 100: 114515.

52 Brabec C, Dyakonov V, Scherf U. Organic Photovoltaics. Wiley-VCH: Weinheim. 2008.

53 Rand BP, Genoe J, Heremans P, Poortmans J. Solar cells utilizing small molecular weight organic semiconductors. Prog Photovolt 2007; 15: 659-676.

54 Ouyang W, Manaka T, Naitou S, Kunitomo K, Iwamoto M. Optical second-harmonic generation in hydrogenated amorphous silicon single- and double-junction solar cells. Jpn J Appl Phys 2012; 51: 070209.

55 Katsuno T, Manaka T, Ishikawa T, Ueda H, Uesugi T et al. Current collapse imaging of Schottky gate AIGaN/GaN high electron mobility transistors by electric fieldinduced optical second-harmonic generation measurement. Appl Phys Lett 2014; 104: 252112.

cc) (i) $\Theta$ This work is licensed under a Creative Commons AttributionBY NC ND NonCommercial-NoDerivs 4.0 International License. The images or other third party material in this article are included in the article's Creative Commons license, unless indicated otherwise in the credit line; if the material is not included under the Creative Commons license, users will need to obtain permission from the license holder to reproduce the material. To view a copy of this license, visit http:// creativecommons.org/licenses/by-nc-nd/4.0/ 